\title{
Multiscale ATUM-FIB Microscopy Enables Targeted Ultrastructural Analysis at Isotropic Resolution
}

Georg Kislinger ${ }^{1}$, Helmut Gnaegi ${ }^{2}$, Martin Kerschensteiner ${ }^{3}$, Thomas Misgeld ${ }^{1}$, Mikael Simons ${ }^{1}$ and Martina Schifferer ${ }^{4}$

${ }^{1}$ TUM/DZNE, Munich, Bayern, Germany, ${ }^{2}$ Diatome, Nidau, Bern, Switzerland, ${ }^{3}$ LMU, Munich, Bayern, Germany, ${ }^{4}$ DZNE, Munich, Bayern, Germany

Volume Electron Microscopy (EM) not only enables dense reconstructions of neuronal circuits but provides the tissue context for targeted high resolution visualization of selected biological structures. Currently, 3D ultrastructure can be solved by destructive techniques including serial block-face electron microscopy (SB-SEM Denk \& Horstmann, 2004; ), focused ion beam-scanning electron microscopy (FIBSEM (Heymann et al., 2006)) or serial sectioning (automated tape-collecting ultramicrotomy ATUM (K. J. Hayworth et al., 2014), transmission electron microscope camera array TEMCA (Bock et al., 2011)) methods. While destructive methods benefit from high alignment accuracy, they lack the option of reacquisition and hierarchical imaging. However, as microtomy-based approaches are limited by their poor $\mathrm{z}$ resolution, ion milling techniques are required if isotropic high resolution voxels are needed. Despite recent advances in the application of alternative milling strategies, targeted FIB-SEM imaging is still required to restrict the acquisition volume. This is mainly achieved by correlated workflows involving targeted trimming guided by endogenous and artificial landmarks (Bishop et al., 2011). X-ray micro computed tomography (microCT) (Bushong et al., 2015) has emerged as a tool for facilitated ROI relocation within the processed EM sample. So far, microCT imaging options are not commonly accessible and the technique only provides a virtual map for subsequent guided destructive sample preparation. An alternative prescreening of embedded tissue at a larger scale is implemented by rendering it accessible to light and electron imaging modalities. Ultrathick sectioning at $20 \mu \mathrm{m}$ by the hot knife method provides samples that are accessible to large-scale FIB-SEM (Kenneth J. Hayworth et al., 2015) enabling seamless reconstruction of large tissue blocks.

We developed a multiscale method for targeted FIB-SEM on semithick (2-10 $\mu \mathrm{m})$ sections named ATUMFIB. Microtomy of semithick sections is facilitated by an optimized resin formulation and a custom-built ultra diamond knife with temperature control. Serial thick sections are collected onto carbon nanotube (CNT) tape (Kubota et al., 2018), compatible with both transmitted light and scanning electron microscopy (SEM). 3D information obtained by serial Light and Electron Microscopy exposes the detailed ultrastructure of regions of interest that are directly accessible for targeted FIB-SEM. While providing direct physical access to isotropic high resolution imaging of multiple ROIs by FIB-SEM this method enables the archiving of 3D tissue context information. We demonstrate the relocation of microglia contact sites with amyloid plaques in a mouse model for familial Alzheimer Disease (FAD). 


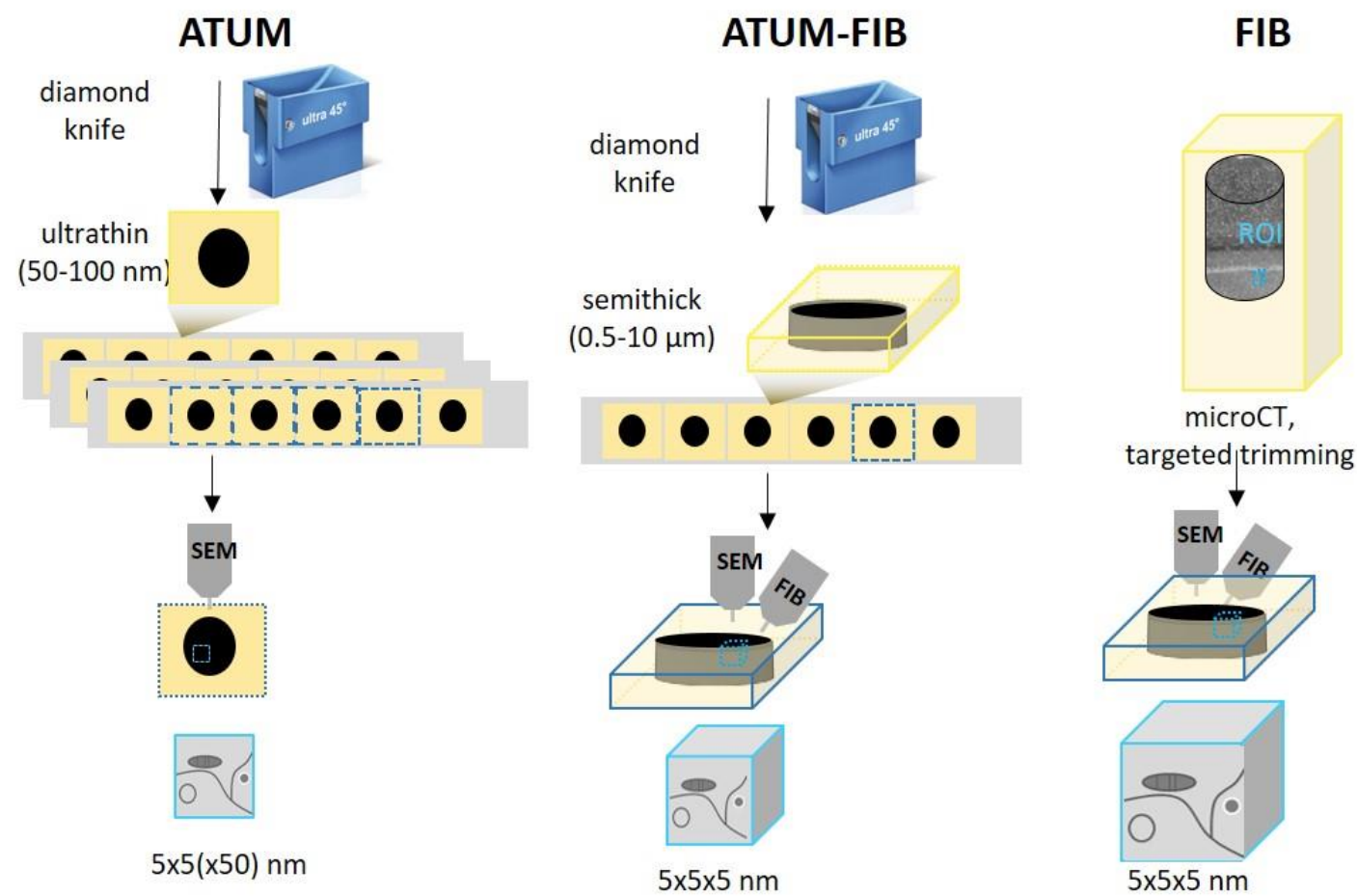

Figure 1. ATUM-FIB is a new technique for targeted volume EM.

A
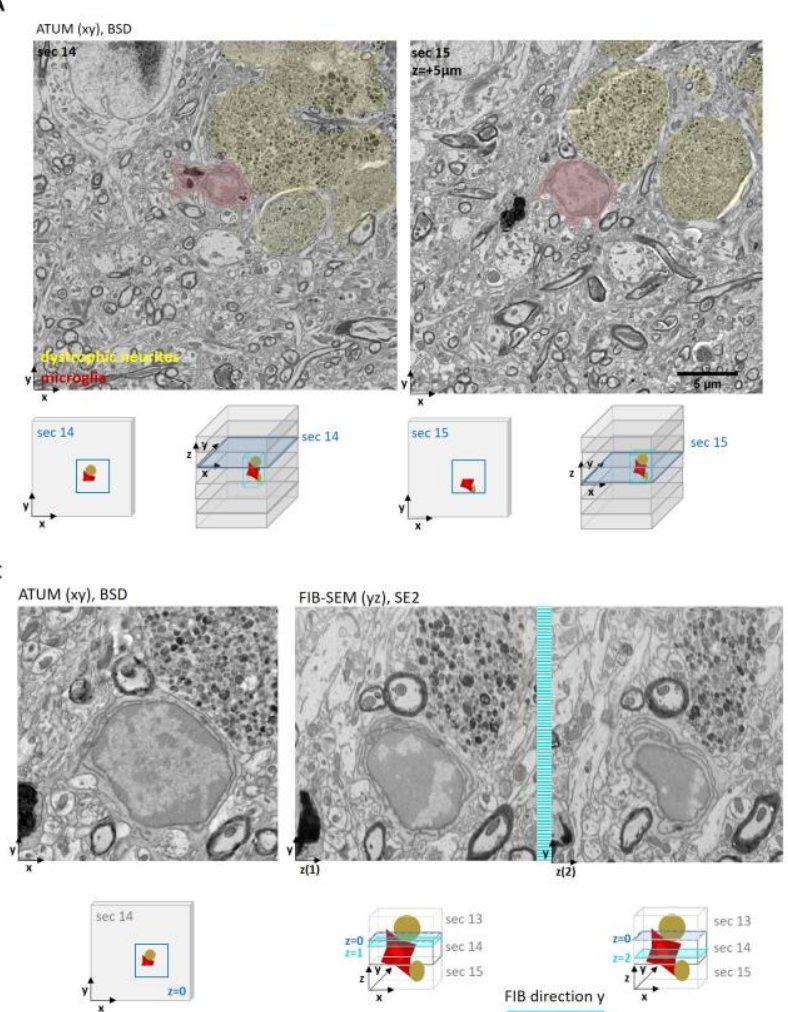

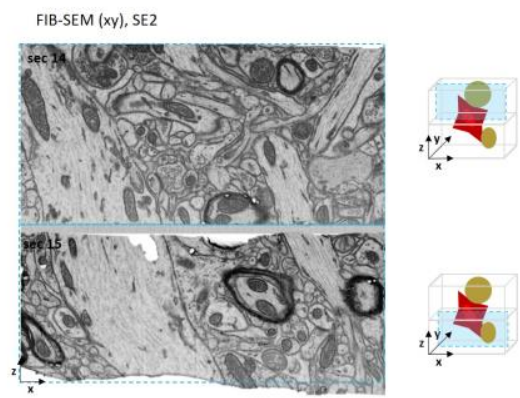

D
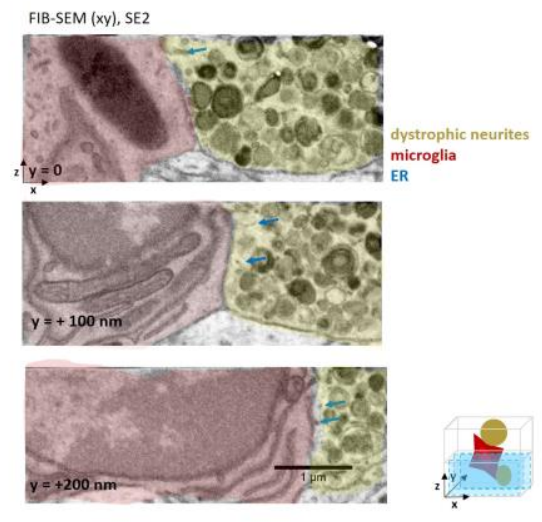

$i^{4}+\frac{1}{x}$

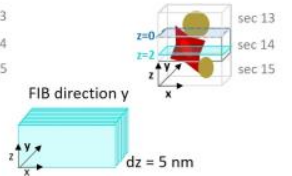

Figure 2. Targeted FIB-SEM on selected consecutive semithick sections of mouse familial Alzheimer Disease (FAD) cortex. 
References

Bishop, D., Nikic, I., Brinkoetter, M., Knecht, S., Potz, S., Kerschensteiner, M., \& Misgeld, T. (2011). Near-infrared branding efficiently correlates light and electron microscopy. Nat Methods, 8(7), 568-570. doi:10.1038/nmeth.1622

Bushong, E. A., Johnson, D. D., Kim, K.-Y., Terada, M., Hatori, M., Peltier, S. T., . . Ellisman, M. H. (2015). X-Ray Microscopy as an Approach to Increasing Accuracy and Efficiency of Serial Block-Face Imaging for Correlated Light and Electron Microscopy of Biological Specimens. Microscopy and Microanalysis, 21(1), 231-238. doi:10.1017/S1431927614013579

Denk, W., \& Horstmann, H. (2004). Serial block-face scanning electron microscopy to reconstruct threedimensional tissue nanostructure. PLoS biology, 2(11), e329-e329. doi:10.1371/journal.pbio.0020329

Djannatian, M., Timmler, S., Arends, M., Luckner, M., Weil, M.-T., Alexopoulos, I., . . . Simons, M. (2019). Two adhesive systems cooperatively regulate axon ensheathment and myelin growth in the CNS. Nature Communications, 10(1), 4794. doi:10.1038/s41467-019-12789-z

Hayworth, K. J., Morgan, J. L., Schalek, R., Berger, D. R., Hildebrand, D. G., \& Lichtman, J. W. (2014). Imaging ATUM ultrathin section libraries with WaferMapper: a multi-scale approach to EM reconstruction of neural circuits. Front Neural Circuits, 8, 68. doi:10.3389/fncir.2014.00068

Hayworth, K. J., Xu, C. S., Lu, Z., Knott, G. W., Fetter, R. D., Tapia, J. C., . . . Hess, H. F. (2015). Ultrastructurally smooth thick partitioning and volume stitching for large-scale connectomics. Nature Methods, 12, 319. doi:10.1038/nmeth.3292

Kubota, Y., Sohn, J., Hatada, S., Schurr, M., Straehle, J., Gour, A., . . Kawaguchi, Y. (2018). A carbon nanotube tape for serial-section electron microscopy of brain ultrastructure. Nature Communications, 9(1), 437. doi:10.1038/s41467-017-02768-7 